\title{
New fat ingredients in confectionary for a healthy diet
}

\author{
Zinaida Baranova, Sergey Nikonovich, Natalia Tarasenko, Elena Baranova, and Svetlana \\ Kucherova \\ Kuban State Technological University, Moskovskaya Street, 2, 350072 Krasnodar, Russian \\ Federation
}

\begin{abstract}
An important segment of the functional food market is products of the confectionery industry covered with chocolate or confectionery glaze for a healthy diet. The selection of high-quality and technological fat ingredients for the glaze for such products is often difficult due to the specific properties of the functional ingredients in the glaze composition. An additional source of concern is the realization that there are a limited number of vegetable oils and fats in nature that have optimal crystallization and melting characteristics. Therefore they must be subjected to modification processes, such as hydrogenation, to form the hardness and main rheological characteristics of the future fat base. Such processes can negatively affect the consumer properties of the ready-made glaze, due to the formation of trans-isomers of fatty acids. A technology for the production of new fat ingredients by a combined method has been developed. On the basis of the obtained cocoa butter substitutes of nontempered mixed type, which do not contain trans-isomers of fatty acids, and have optimal characteristics of crystal formation, experimental batches of confectionery glaze were produced and a set of indicators that form the consumer properties of the ready-made product was investigated. The result of the study was practical recommendations for the use of confectionery glazes based on the developed fat ingredients.
\end{abstract}

\section{Introduction}

According to a comprehensive study of the consumer market of confectionery products, including items covered with chocolate or confectionery glazes, the majority of consumers of different population groups see a direct connection between health and well-being with their traditional diet. In modern conditions the importance of the intention to possess the most important basic value of life-health, without resorting to drugs-is only increasing.

The rational diet is necessary to keep health, because food is the main source of biologically active substances $[1,2]$.

The confectionery products, including items covered with chocolate or confectionery glaze should be one of the important segments of the market of functional food products of the XXI century.

The use of vegetable raw materials-oils and fats is considered to be the most effective and at the same time cost-effective ways to increase the nutritional value of confectionery 
products and create functional food products that are useful for health. It is necessary to study the system of fat ingredients included in the composition of glazing coatings.

Cocoa butter is the most expensive between all ingredients included in the chocolate glaze. It can be considered a perfect fat ingredient. All manufacturers of specialized fats for the confectionery industry take it as a sample. Its properties are based on the physical and chemical characteristics of all other alternative fats.

The specific fatty acid composition is the main reason for choosing cocoa butter as a sample fat. The largest amount in the composition is accounted for by three fatty acids: palmitic, stearic and oleic. The triglyceride composition is also unique, because no other known vegetable oils and fats contain a similar ratio of basic symmetric triglycerides (POP, POS, SOS) [3].

The technological scheme of processing cocoa beans and the level of competence of the staff play an important role in the processing of this raw material [4]. Trends of sharp changes in the pricing policy in the cocoa butter market, combined with the inability to guarantee a high and stable yield of this vegetable oil from year to year, lead to an increase in the cost of fat ingredients created on the basis of cocoa butter and reduce their availability and prevalence of use; limit the range of chocolate-glazed confectionery products.

The investigations are relevant aimed at creating new fat ingredients - various types of alternatives to cocoa butter (cocoa butter equivalents and substitutes), containing natural and modified vegetable oils and fats of different degrees of hardness, subject to various complex modifications to expand their fields of application.

The modern market of food products containing vegetable fat ingredients in their composition is faced with a number of legal restrictions based on the requirements of consumers.

The growing demand for more healthy and high-quality confectionery fat-containing products should be included. From 01.01.2018, the requirements of TR CU (Technical Regulations of the Customs Union) 024/2011 "Technical Regulations for fat-and-oil products" became effective, indicating that manufacturers should normalize the transisomers of fatty acids in confectionery fats to $2 \%$.

Until recently, this regulation is not related to cocoa butter substitutes of non-regulated non-lauric and mixed types. Since 01.01.2026 according to the draft amendments to TR CU 024/2011 a similar restriction will be established for the alternatives of cocoa butter.

The development of new, combined methods for the production of fat ingredients for the confectionery industry and the improvement of the processes of modification of oils and fats are modern trends in food production [5].

The comprehensive study of the physico-chemical, structural and mechanical properties of the developed new fat ingredients and the study of the rheological characteristics of confectionery glazes were carried to develop investigation about combined methods for the production of ingredients for the confectionery industry in order to create new fat ingredients-non-moderated mixed-type CBS without trans-isomers of fatty acids in the composition and optimal properties of crystal formation.

\section{Materials and methods}

During analytical research modern conventional and special measuring methods are used to evaluate the quality of raw materials and ready-made products.

The construction of the cooling rate curve for the developed fat ingredients and the calculation of the pour point were carried out by the Jenson method at a temperature of +17 ${ }^{\circ} \mathrm{C}$. Determination of the degree of the developed fat ingredients shrinkage was analyzed according to the developed method. 
The content of the mass fraction of solid triglycerides at six different temperatures was determined by pulsed nuclear magnetic resonance on the devices «Chromatek Proton 20M» and «MQ20» of the company «BRUKER». The triglyceride composition of the fat ingredients was investigated by liquid chromatography on the «Agilent 1260 Infinity LC» chromatograph. The determination of the hardness of confectionery glazes was carried out on a structurometer «BROOKFIELD STZ $4500 »$. The rheological characteristics of the studied confectionery glazes (plastic viscosity, yield point) were measured by the Casson method on a BROOKFIELD DV2T rotary viscometer at a temperature of $+40^{\circ} \mathrm{C}$, in the range of shear rates from $5 \mathrm{~s}-1$ to $60 \mathrm{~s}-1$. The Buhler BCI crystallization coefficients were determined on a Buhler Multitherm device.

\section{Results and discussion}

The developed technology for the production of non-tempered mixed-type CBS (Cocoa Butter Substitute) is combined, and includes well-known methods for modifying vegetable oils and fats. At the same time the samples contain a minimum of trans-isomers of fatty acids according to the current trends in the field of healthy nutrition and legislative requirements [6]. The first stearic fraction of palm kernel oil is included in the fat composition subjected to a full cycle of hydrogenation in the amount of 5\% (CBS-2) and $10 \%$ (CBS-3) of the mass fraction of the fat phase, which allows the formation of a triglyceride composition with a significant amount of POP (Fig.1).

\section{Triglyceride composition, \%}

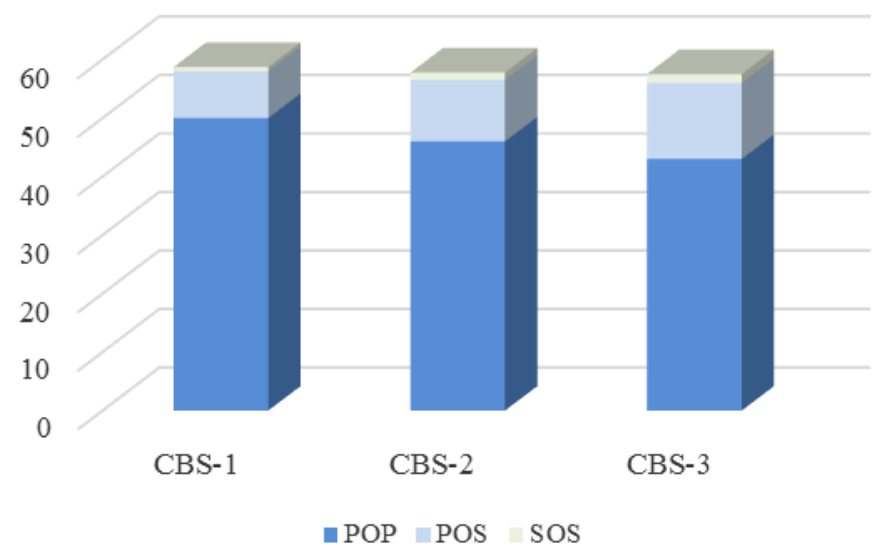

Fig. 1. Mass fraction of the main triglycerides developed by CBS $5 \%$.

The content of lauric acid in the composition of the new fat ingredients does not exceed

The complex triglyceride composition of the developed CBS makes it possible to obtain a confectionery glaze, which provides a bright disclosure of the taste of the glazed confectionery, melting with a light cooling effect and excellent storage capacity. 


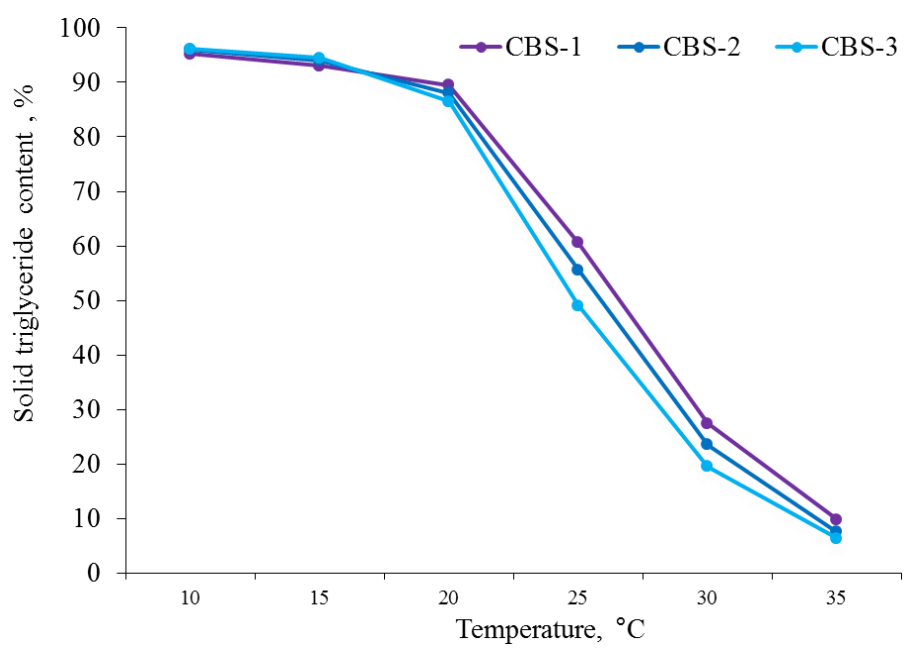

Fig. 2. Mass fraction of STG developed by CBS

Confectionery glaze should have a combination of structural and mechanical properties that allow the glazing coating to have high values of the mass fraction of solid triglycerides (STG) in the temperature range from $+20^{\circ} \mathrm{C}$ to $+25^{\circ} \mathrm{C}$ and at the same time completely melt at a temperature of $+35^{\circ} \mathrm{C}$ (close to the temperature of the human body). This means that a specific melting profile is required (Figure 2).

The developed new fat ingredients have good thermal stability and a sharp melting profile. This is ensured by the presence of solid fat in the composition: a complex of vegetable oils subjected to fractionation.

It is known [7] that the general trend for cocoa butter alternatives, including mixed-type SMC, with a low content of trans-isomers, is a lower crystallization rate than for fats with a high content of trans-isomers. And the lower the content of trans isomers, the lower the rate of solidification, even with the introduction of emulsifiers-crystal-forming agents. Therefore a great attention should be paid to the research of the melting and solidification characteristics of the developed fat ingredients.

During crystallization, fats have the property of decreasing in volume, which provides the selection of confectionery glaze in tiles and products from molds [8]. To adjust the cooling modes of confectionery glaze, it is necessary to understand the complex properties of fat ingredients that characterize the crystallization processes: the degree of shrinkage and the Buhler crystallization index (the higher the value of the BCI coefficient, the higher the crystallization rate [9].

The values of the BCI shrinkage rate of the developed fat ingredients are shown in Table 1 .

Table 1. Degree of shrinkage of the developed CBS.

\begin{tabular}{|c|c|c|c|}
\hline \multirow{2}{*}{ Sample name } & \multicolumn{2}{|c|}{ Degree of shrinkage at temperature } & \multirow{2}{*}{ BCI } \\
\cline { 2 - 3 } & $+2^{\circ} \mathrm{C}$ & $+4^{\circ} \mathrm{C}$ & 8.6 \\
\hline CBS-1 & 10.20 & 11.12 & 8.3 \\
\hline CBS -2 & 9.00 & 10.98 & 8.5 \\
\hline CBS -3 & 11.78 & 12.20 & \\
\hline
\end{tabular}

Sample CBS -3 , characterized by a high content of lauric acid $-4.66 \%$, has the highest shrinkage both at $+2{ }^{\circ} \mathrm{C}$ and at $+4^{\circ} \mathrm{C}$.

Additionally, the study of the crystallization rate of CBS samples using a structurometer showed that the samples of CBS-2 and CBS-3 have a higher crystallization rate, which is 
consistent with the previously obtained data on the crystallization of these fat ingredients (Fig. 3).

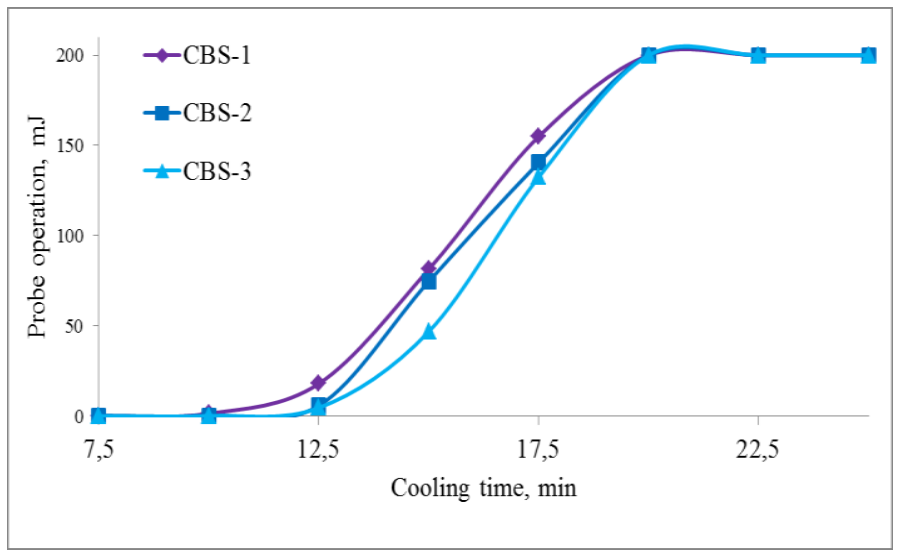

Fig. 3. Crystallization rate of CBS samples

On the basis of the prepared samples of fat bases, the confectionery glaze was developed according to the traditional recipe. The resulting samples of confectionery glaze are assigned names similar to the names of the corresponding samples of fat ingredients.

It is not uncommon when several samples of confectionery glaze have the similar viscosity values and different pour point values and they affect the productivity and technological parameters of the glazing equipment in various ways.

The recommended values of rheological characteristics for confectionery glaze are $1.0-$ 3.0 $\mathrm{Pa} \times \mathrm{s}$ for the plastic viscosity, 7.0 - 12.0 Pa for the yield point [10].

The measurement values obtained on the rotary viscometer made it possible to calculate the rheological parameters by graphic analytic method for each sample of confectionery glaze. The results were obtained on the yield point and plastic viscosity of the studied glaze samples (table 2).

Table 2. Data on the rheological parameters of the developed CBS.

\begin{tabular}{|c|c|c|}
\hline \multirow{2}{*}{ Name sample } & \multicolumn{2}{|c|}{ Rheological characteristics } \\
\cline { 2 - 3 } & Yield point, Pa & Plastic viscosity, $\mathrm{Pa} \times \mathrm{c}$ \\
\hline CBS-1 & 9.47 & 1.17 \\
\hline CBS -2 & 9.56 & 1.15 \\
\hline CBS-3 & 9.85 & 1.10 \\
\hline
\end{tabular}

The obtained characteristics of the rheological properties of confectionery glazes showed that the developed fat ingredients will provide a "light" coating of the glaze on the ready-made product with a sufficiently thin and uniform layer, while the confectionery glaze will be sufficiently viscous to prevent breaks in the glaze layer on the product and maintain a given layer thickness.

\section{Conclusions}

At the same time, work is actively carried out to introduce regulatory restrictions on the number of trans-isomers of fatty acids for all types of cocoa butter alternatives, including mixed-type CBS. 
1. The developed technology of production of cocoa butter substitute of non-tempered mixed type is combined and only well-known methods of modification of vegetable oils and fats are included.

2. The new fat ingredients are recommended as a complete replacement for expensive cocoa butter, while the samples are characterized by the absence of trans-isomers of fatty acids and do not require tempering.

3. The triglyceride composition of the new fat ingredients differs in a significant amount of POP $48.0-50.0 \%$, ROS - up to $13.0 \%$ and SOS - up to $2.0 \%$. It provides a sharp melting profile and good thermal stability. CBS samples will provide the confectionery glaze with organoleptic properties as close as possible to the glaze based on cocoa butter, extend the shelf life, due to less susceptibility to recrystallization.

4. CBS samples have excellent crystallization characteristics: BCI values from 8.3 to 8.6 and the degree of shrinkage from $9.0 \%$ to $12.0 \%$ are established. These values of the main cooling parameters are possible to optimize the glazing process and line performance due to faster crystallization.

5. The obtained confectionery glazes based on the developed CBS are recommended for glazing a wide range of confectionery products: praline and candies «Assorti», fondant varieties of sweets, and caramel. Thus, the use of the developed CBS for glazing give opportunity to choose the best option for a particular confectionery product, taking into account the peculiarities of its production.

As a result of a comprehensive assessment of the results of the research, it can be concluded that the developed fat ingredients (CBS-1, CBS-2, CBS-3) are technological and recommended for use in the production of confectionery glazes with different consumer characteristics (hardness, reduction, viscosity), which reflects the ability to obtain a product with technological indicators and make it possible to solve a specific production task.

\section{References}

1. L. Donchenko, V. Nadykta, Food safety, 528 (2001)

2. Hou-Pin Su, Chuang-Ping Lien, Tan-Ang Lee, Jou-Hsuan Ho, Science of food agriculture, 90 (2010)

3. K. Rajah, Fats in food technology, 221 (2016)

4. I. Korolev, Oils and fats, 01, 20 (2019)

5. N. Tarasenko, I. Krasina, Z. Baranova, N. Tretyakova, Research Journal of Pharmaceutical, Biological and Chemical Sciences, 7, 1083 (2016)

6. I. Korolev, Oils and fats, 03-04, 10 (2020)

7. R. Hartel, J.H.von Elbe, R. Hofberger, Confectionery science and technology, 732 (2019)

8. Z. Baranova, Development and evaluation of consumer properties of fat mixtures for confectionery glazes, 10 (2020)

9. V.G. Lobanov, Y.I. Slepokurova, I.M. Zharkova, T.N. Koleva, Y.F. Roslyakov, A.P. Krasteva, Foods and Raw Materials, 6 (2) (2018)

10. N.V. Leyberova, O.V. Chugunova, N.V. Zavorokhina, Economy of Region, 4 (2011) 